\title{
Therapeutic Effects of Low-Level Laser on Lateral Epicondylitis from Differential Interventions of Chinese-Western Medicine: Systematic Review
}

\author{
Wen-Dien Chang, Jih-Huah Wu, ${ }^{2}$ Wen-Ju Yang, and Joe-Air Jiang ${ }^{3}$
}

\section{Abstract}

Background: Low-level laser therapy (LLLT) is a conservative treatment for lateral epicondylitis (LE), but it is also an alternative intervention between the very different approaches of Chinese and Western medicine. Objective: The purpose of this study was to systematically review and meta-analyze the therapeutic effects of

AU4 LLLT on LE. Methods: We searched several electronic databases, including Medline, PubMed, and CINAHL, and explored studies that were randomized controlled trials on the therapeutic effects of LLLT on LE from 1990 to February 2009. These studies were systematically reviewed for the difference in therapeutic effects among various LLLTs on acupuncture points and on tender and myofascial trigger points (MTrPs). The reviewed therapeutic effects included pain, grip strength, range of motion (ROM), and weight tests, and were compared by meta-analysis. Results: We selected ten articles, and in seven of them the irradiation was conducted on tender points or MTrPs in the experimental groups. In two other articles, the irradiation was conducted on acupuncture points, and the last one was conducted on both kinds of points. Only three articles provided sufficient data for meta-analysis. The results revealed that applying LLLT on tender points or MTrPs is an effective means to improve the effect size (ES) of pain release after treatment (pooled ES: 0.71, 95\% CI: 0.82- 0.60) and follow-up (pooled ES: 1.05, 95\% CI: 1.16- 0.94). LLLT application was also able to increase the grip force, ROM, and weight test $(p<0.05)$. Conclusions: We suggest that using LLLT on tender points or MTrPs of LE could effectively improve therapeutic effects.

\section{Introduction}

L ATERAL EPICONDYLITIS (LE), also known as tennis elbow, is a commonly seen musculoskeletal disease in the dominate hand stemming from overuse or repeated wrist movements. Clinical symptoms include tender points, myofascial trigger points (MTrPs), and pain induced in the lateral epicondyle or muscles attached to forearm when using the arms, especially when grasping and lifting heavy objects. ${ }^{1}$ The diagnosis of LE is usually confirmed by provoking tests, including resistive contraction of wrist or finger extensors, to reproduce or aggravate clinical symptoms. These symptoms can affect functional movements and activities and can last for 3 to 6 years. $^{2}$ Therefore, most patients receive treatments during the first 6 to 24 months. ${ }^{3}$ Treatment strategies include steroid injections, non-steroidal anti-inflammatory drugs (NSAIDs), Chinese medicine, and physical therapy. ${ }^{1}$
A common suggestion among Chinese and Western practitioners for acute LE is to rest immediately, to stop all painful activities, and to reduce inflammation with an ice pack. In traditional Chinese medicine (TCM), dialectical thinking and approach would suggest using acupuncture and external ointments. An example is acupuncture at Shousanli (LI10) and Quchi (LI11) points, followed by an external plaster to alleviate heat in order to release pain and to promote blood circulation and to remove blood-stasis. ${ }^{4}$ In the Western physical therapy approach, hot packing, modality, orthosis, and kinesiotaping of the elbow are normally recommended. ${ }^{1}$ Modality approaches include electrical stimulation, ultrasound, and low-level laser therapy (LLLT). ${ }^{5}$ TCM therapy may also include local massage and acupuncture accompanied by herbs providing the effects of warming channels to dredge collaterals, promoting blood circulation to remove bloodstasis, and improving qi flow. ${ }^{4}$ Based on the pain-relieving

\footnotetext{
${ }^{1}$ Department of Rehabilitation Medicine, Da Chien General Hospital, Department of Bio-Industrial Mechatronics Engineering, National Taiwan University, Taiwan.

${ }^{2}$ Department of Biomedical Engineering, Ming Chuan University, Taiwan.

${ }^{3}$ Department of Bio-Industrial Mechatronics Engineering, National Taiwan University, Taiwan.
} 
theory of acupuncture, finding the appropriate treatment points on the channels could consequently bring about the release of endorphins and an increase in 5-hydroxy tryptophan in the brain. ${ }^{4}$ In addition, a TCM explanation for the effects of acupuncture is that qi or vital energy flows elicited by needling can remove energy-stagnation and then promote a balanced energy system to achieve pain-relieving effects. ${ }^{6}$ Symptoms of LE can bring about MTrPs in the wrist and finger extensors. Melzack et al. indicated that the correlation between MTrPs and the corresponding acupuncture points was $71 \%{ }^{6}$ However, Hong and Simons ${ }^{7}$ suggested that the etiology of MTrPs was related to sensitized nociceptors and dysfunctional endplates, and that the MTrPs were not the same as acupuncture points. Some "MTrPs" are actually "Ashi points" in acupuncture, but some "Ashi points" are not MTrPs. ${ }^{8}$ Moreover, tender points caused by LE were also found to be similar to Ashi points. ${ }^{9}$ However, Simons et al. ${ }^{10}$ proposed that MTrPs were different from tender points. Pressing on tender points could induce local, but not referred, pain while pressing on MTrPs could cause referred pain and local twitch response. LLLT has been applied in laser acupuncture, which has the benefits of being painless, sterile, safe, dose controllable, and easy to operate. ${ }^{11}$ In summary, we examined three choices when applying LLLT on LE: emitting on acupuncture points, tender points, and MTrPs.

In LLLT, a low dose is usually used to induce photobiochemical effects without causing significant changes in tissue level. Previous studies have shown that LLLT could promote the synthesis of prostaglandin and consequently reduce pain. ${ }^{12,13}$ Furthermore, arachidonic acid would be transported into endothelial and smooth muscle cells to enhance dilation of blood vessels and reduce inflammation. ${ }^{14}$ Such laser irradiation would not burn or damage tissue, but would change tissue metabolism through a process called "Photobiomodulation" ${ }^{15}$ Some evidence derived from animal studies showed that LLLT could promote collagen fiber formation and as a result facilitate tendon repair. ${ }^{16}$ However, collagen fiber formation could be obviously affected by factors such as dosage and wavelength of the laser. ${ }^{16}$ Some studies also found that a power density more than $100 \mathrm{~mW} / \mathrm{cm}^{2}$ might adversely inhibit the activity of fibrous tissue and the synthesis of collagen fiber. ${ }^{17,18}$ In summary, LLLT could cause changes in biochemistry in cells and tissue through photobiomodulation, and these changes could be affected by different dosage and wavelength of laser.

In the past, the most appropriate model explaining photobiomodulation was the Arndt-Schultz law, which stated that a certain level of laser energy is required to reach the threshold and hence trigger biological stimulation. ${ }^{12}$ Different media of laser irradiation result in different depths of penetration and various effects. Although in some systematic reviews, it had been substantially discussed that various clinical doses, methods, and modes of LLLT could bring about different treatment effects, ${ }^{19,20}$ there are still very few papers investigating the different effects of Western and Chinese medicine. Therefore, the aim of this systematic review and meta-analysis study was to investigate the effectiveness of applying LLLT on LE in both traditions. The discussed effectiveness includes pain of elbow, grasp force, and other related measurements. These may in turn benefit both Western and Chinese medicine therapies.

\section{Materials and Methods}

This study searched papers published between January of 1990 and February of 2009 through Medline, PubMed and CINAHL. Key words included low-level laser, lateral epicondylitis, tennis elbow, and laser acupuncture. The selected articles were also utilized to assess other related studies.

Because this systematic review and meta-analysis study was designed to understand the effectiveness of LLLT on LE, only studies of randomized controlled trials (RCTs) were included that met the following criteria: (1) the subjects were diagnosed as having LE of elbow with pain induced by resisted extension of the wrist, (2) LLLT was used on the inflamed tendons, MTrPs, or acupuncture points as a treatment of LE, (3) the study must have involved randomized grouping with single- or double-blind design, and (4) the control group must have received a non-laser or placebo laser treatment with zero output. Based on these inclusions, 10 studies were selected. ${ }^{21-30}$ Their quality was rated according to Physiotherapy Evidence Database scale (PEDro) guidelines. ${ }^{31}$

Methods of evaluation used in related studies were chosen to assess pain, muscle power, function, and effectiveness after applying LLLT to LE.

(1) Pain measurement: This was to assess the effectiveness of treatment by observing subjects' movements and asking them to grade their pain under natural conditions or during press palpation. Based on past experience, subjects quantitatively expressed their present pain according to the most commonly used Visual Analogue Scale (VAS) with 0 for no pain and 10 for the worst possible pain. Another method was to rate pain by Five Point Verbal (FPV) scale, in which 0 represents no pain, 1 represents mild pain, 2 represents moderate pain, 3 represents severe pain, and 4 represents very severe pain.

(2) Grasp or finger grip force: This was measured by a grip dynamometer or a vigorimeter to assess the grip force of the hand. A pinch dynamometer was also used to measure the pinch force of the fingertips, to represent muscle strength during functional movements.

(3) Muscle test: Subjects were given resistive tests of forearm movements, such as pronation, supination, wrist extension and middle finger test, to see if any symptom was induced. The Mercy Wedge Pro (MWP) exercise was also used to assess pain during wrist extension. Weight tests ranging from 1 to $4 \mathrm{~kg}$ served as a means of measurement to quantify the weight induced symptoms when straightening the wrist. Another test to quantify muscle strength was the manual muscle testing (MMT) which rates muscle strength with six grades.

(4) Range of motion (ROM) of the wrist joint: The painless range of motion of wrist joint was measured by a goniometer.

(5) Pain pressure threshold (PPT): Tender points were assessed quantitatively by applying a pressure algometer directly on muscles until the pain threshold is determined.

(6) Questionnaire and treatment effects: Upper extremity function was assessed by the Disabilities of the Arm, Shoulder and Hand questionnaire (DASH), which in- 
cludes 30 questions with responses ranging from one point to five points. Subjects with higher scores may be more severely affected by LE. Follow-up assessments of the effectiveness of treatments were obtained by asking the subjects to subjectively report their general health status rated as excellent, good, improved, slightly improved, unchanged, or worse.

\section{Statistical analysis}

Data obtained from the selected literature were numbered, entered into datasheets, and divided into three parts. The first part, as shown in Table 1, summarizes the features of each study, including the representative author and publication date, number and average age of subjects, sites of treatments, laser parameters, treatment dosages, and quality of the study. The second part, as shown in Tables 2 and 3, includes periods and times of investment, assessable items and time, and the results of the assessments. In the third part, results in terms of effectiveness, expressed in average values and standard deviation, or the $95 \%$ confidence interval (CI) providing the standard deviation, were further selected to conduct an integrated comparison. Meta-analysis was then used to calculate the individual and total effect size (ES) for each variable. ${ }^{32}$

Pooled standard deviation (pooled SD) for each variable was calculated by the equation: pooled $\mathrm{SD}=$ $\sqrt{\left[\left(n_{1}-1\right) s_{1}^{2}+\left(n_{2}-1\right) s_{2}^{2}\right] /\left(n_{1}+n_{2}-2\right)}$. In this equation, $n_{1}$ and $n_{2}$ respectively represent the number of participants in the experimental and the control groups, while $s_{1}$ and $s_{2}$ represent the SDs obtained from the experimental and the control groups, respectively. Afterwards, the ES for each variable was computed using the equation: $E S=\left(m_{1}-m_{2}\right)$ / pooled $S D$, in which $m_{1}$ and $m_{2}$ respectively represent the average values in the experimental and the control groups. The pooled ES for each variable was then calculated from all the ESs weighted by the number of total subjects. A positive pooled ES indicates a measured positive effect; whereas a negative value indicates that the treatment was ineffective.

The ESs of all assessable items were represented by using $95 \% \mathrm{CI}$, and analyzed statistically by distinguishing zero in the interval. A CI including zero represented that the null hypothesis could not be rejected and no statistically significant difference in effectiveness existed $(p>0.05)$. A positive interval of CI, exclusive of 0 , indicated a statistically significant effectiveness $(p<0.05))^{32}$

According to the criteria of ES proposed by Cohen's $d$, an ES of 0.2 would be defined as small, 0.5 as medium, and 0.8 as large. ${ }^{32}$ Finally, as shown in Table 4 , the estimated values for effectiveness were analyzed and discussed based on the guidelines from the Philadelphia panel classification system, and the grade of recommendation would be determined. ${ }^{33}$

\section{Results}

This study aimed to investigate the effectiveness of applying LLLT on LE in Western and Chinese medicine. Ten papers studying clinical effectiveness with quality assessment scores ranging from 3 to 8 , as shown in Table 1, were selected through our search. ${ }^{21-30}$ Seven of them were studies of comparisons between laser on tender points and laser on 
PHO-2009-2558-Chang_1P.3D 09/17/09 6:28pm Page 4

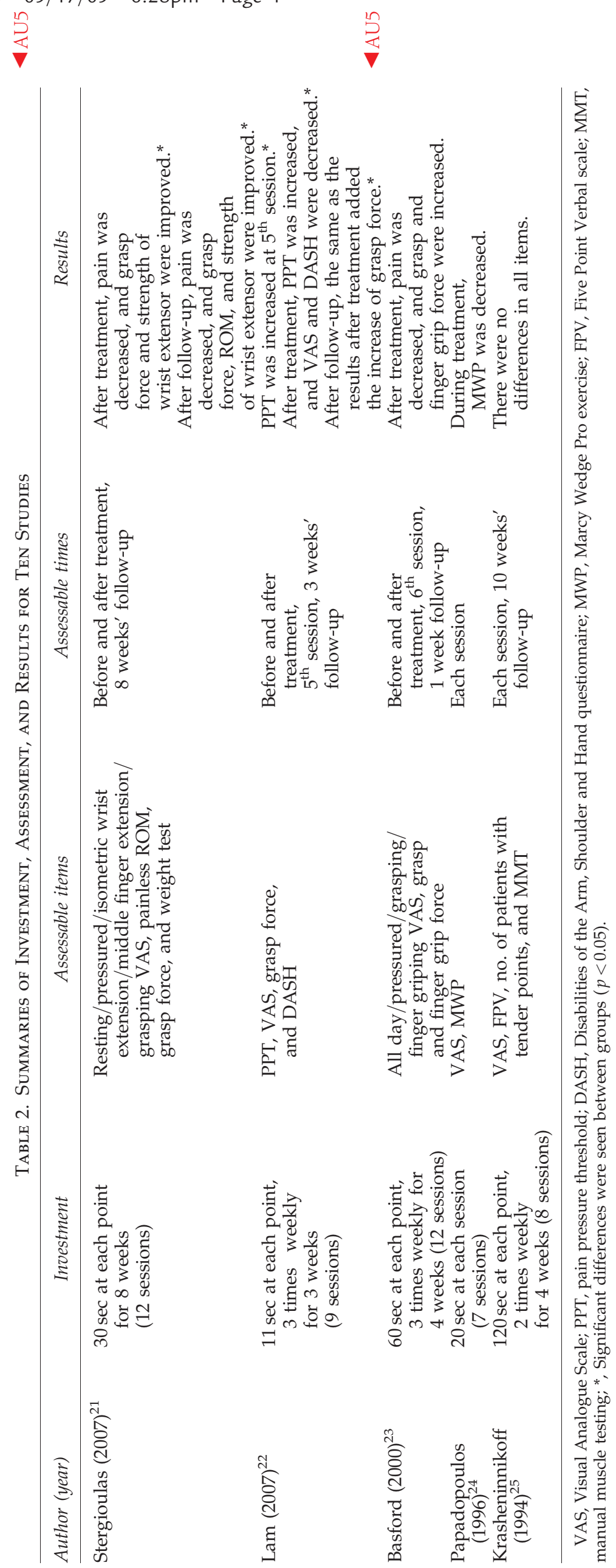


PHO-2009-2558-Chang_1P.3D 09/17/09 6:28pm Page 5

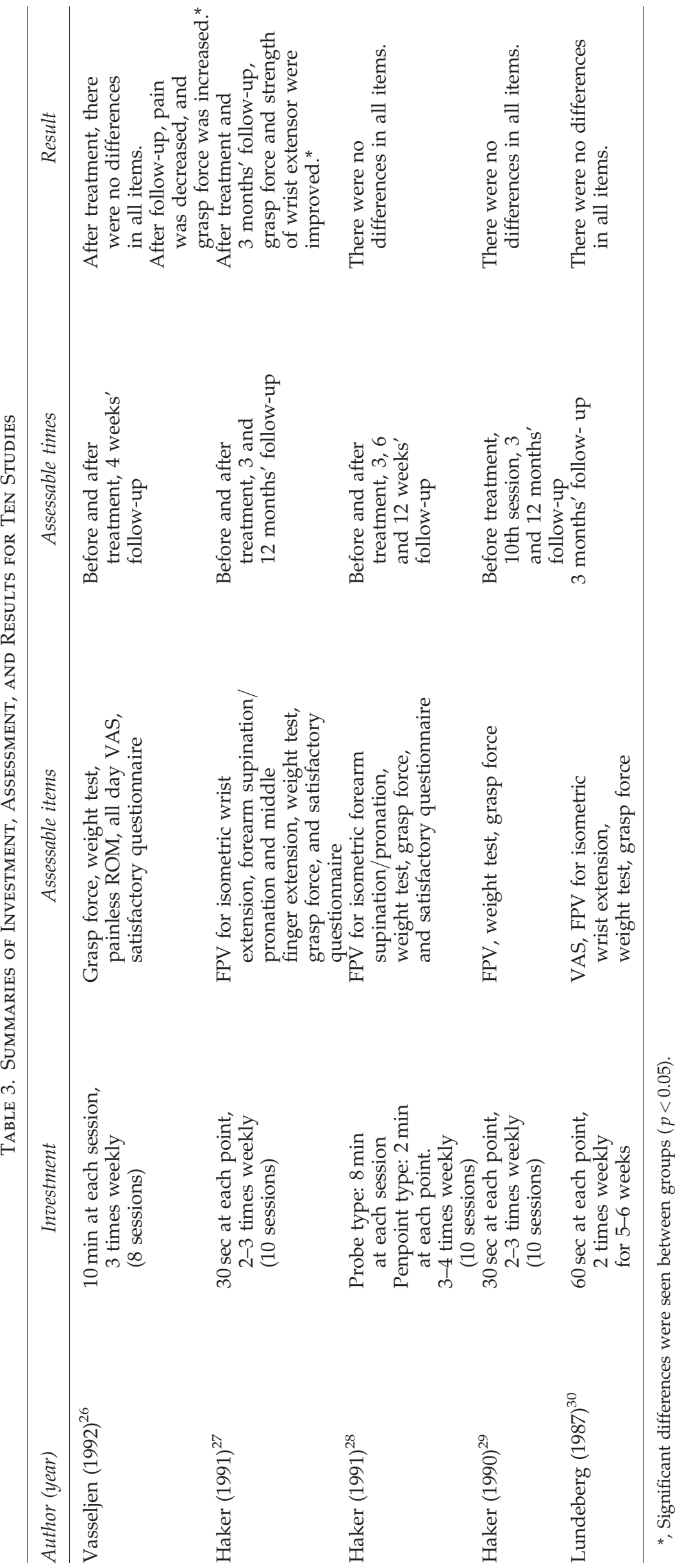


Table 4. Philadelphia Panel Classification System ${ }^{33}$

\begin{tabular}{lllc}
\hline Grade & \multicolumn{1}{c}{ Study design } & Clinical importance & Statistical significance \\
\hline A & RCT (single or meta-analysis) & Important & + \\
B & CCT (single or meta-analysis) & Important & + \\
C + & RCT or CCT (single or meta-analysis) & Important & - \\
C & Any study design & Unimportant & NA \\
D & Well-designed RCT $>100$ patients & Unimportant & - \\
\hline
\end{tabular}

$\mathrm{RCT}$, randomized controlled trial; CCT, controlled clinical trial; +, statistical significance; -, not significant; NA, not applicable (clinical importance is not met, regardless of statistical significance).

the epicondyle of elbow, and all subjects were diagnosed according to Western medical criteria. ${ }^{21-27}$ In these studies, the locations of irradiation were tender points of tendons or muscles. Only Lam and Cheing ${ }^{22}$ utilized MTrPs as points of irradiation. In two studies (Stergioulas, ${ }^{21}$ Lam and Cheing ${ }^{22}$ ), the experimental and the control groups were both given therapeutic exercise. In two other papers, the points of irradiation were acupuncture points of TCM, and the subjects were treated by laser acupuncture. ${ }^{29,30}$ Among all selected papers, only one had conducted laser on both tender points and acupuncture points. ${ }^{28}$

The wavelengths in these 10 papers were $904 \mathrm{~nm}$ in six of them, ${ }^{21,22,26-29} 1.06 \mu \mathrm{m}$ by Basford et al., ${ }^{23} 820 \mathrm{~nm}$ by Papadopoulos and Smith, ${ }^{24}$ and $830 \mathrm{~nm}$ by Krasheninnikoff et al. $^{25}$ In addition, Lundeberg et al. tested lasers with wavelengths of $904 \mathrm{~nm}$ and $632.8 \mathrm{~nm}$ in the study group and compared the results with the control group. ${ }^{30}$ Among these studies, the GaAs diode laser was mostly used with average power ranging from 0.07 to $50 \mathrm{~mW}$. Only Basford et al. ${ }^{23}$ and Lundeberg et al. ${ }^{30}$ used continuous wave irradiation, whereas other authors used pulsed lasers with frequencies between $50 \mathrm{~Hz}$ and $5000 \mathrm{~Hz}$.

As for the dosage of treatment, some previous researches had provided laser treatment dose in terms of joules per point without describing the radius of the probe head and, therefore, joules per $\mathrm{cm}^{2}$ could not be obtained..$^{25,27-30}$ Seven studies with Western medicine diagnosis and treatment had provided the treatment energy between 3.5 and $24 \mathrm{~J} / \mathrm{cm}^{2}$ and dose between 0.36 and $36 \mathrm{~J}^{21-27}$ Another three studies utilizing laser acupuncture had LLLT with dose from 0.004 to $0.9 \mathrm{~J}$ per point. ${ }^{28-30}$

The period and times of investment that these ten papers AU5 had conducted LLLT were summarized in Tables 2 and $3{ }^{21-30}$ Tender points were irradiated for 20 seconds to 8 minutes, ${ }^{21,23-28}$ and acupuncture points were irradiated for 30 seconds to 2 minutes. ${ }^{28-30}$ The times of investment were

AU5 between 7 and 12 sessions. ${ }^{21-30}$ In most of the studies, the evaluations were conducted immediately after treatment and at follow-up from 3 weeks to 12 months. ${ }^{21-29}$ One exception was the study by Lundeberg et al., ${ }^{30}$ in which only a 3 month follow-up evaluation was undertaken with no immediate evaluation after treatment.

All ten papers had assessed resting, motion-induced, and resisted pain. Regarding the pain assessments, seven papers utilized VAS, ${ }^{21-26,30}$ five papers used FPV, ${ }^{25,27-30}$ and two, Krasheninnikoff et $\mathrm{al}^{25}$ and Lundeberg et al., ${ }^{30}$ used both methods. Six papers included weight tests to assess the strength of wrist extensors. ${ }^{21,26-30}$ Papadopoulos and Smith ${ }^{24}$ adopted MWP to assess wrist extensors, while Krashenin- nikoff et al. ${ }^{25}$ utilized MMT to record the strength of wrist extensors. Stergioulas ${ }^{21}$ and Vasseljen et al. ${ }^{26}$ further assessed painless range of motion of wrist joints.

Lam and Cheing ${ }^{22}$ included PPT and the DASH questionnaire. Vasseljen et al. $^{26}$ and Haker and Lundeberg 27,28 assessed effectiveness immediately after treatment and follow-up for a period ranging from 3 weeks to 12 months. Among all the assessment data, Stergioulas, ${ }^{21}$ Lam and Cheing, 22 and Vasseljen et al. ${ }^{26}$ provided average values and standard deviations after treatment and at follow-up. Since these three studies had four measurements in common, the pain scores of VAS, the measurements of grasp force, weight tests, and range of motion of wrist joints, these items were pooled into a meta-analysis. Other papers were individually reviewed and discussed. ${ }^{23-25,27-30}$

\section{The immediate effects of LLLT on pain relief after treatment}

The lower the scores were, the better the effects were. Therefore, the ES values and the null hypothesis of all the pain scales were considered to be negative values. The effects of LLLT on pain relief for 119 subjects in three studies, as indicated in Table 5, were favorable. ${ }^{21,22,26}$ Although the results by Vasseljen et al. ${ }^{26}$ did not show statistically significant effects (95\% CI: $-0.63 \sim 0.08, p>0.05$ ), the pooled ES after being weighted was -0.71 , which was considered to be a medium level effect $(0.8>\mid$ pooled ES $\mid>0.5)$. Furthermore, the $95 \%$ CI did not include zero (95\% CI: $-0.82 \sim-0.60)$ and indicated a statistically significant effect $(p<0.05)$.

\section{The effects of LLLT on pain relief after follow-up}

The ESs of pain relief brought about by LLLT at follow-up, as indicated in Table 6, were obtained from the results of three papers including 119 subjects. ${ }^{21,22,26}$ There was a trend showing that the favorable effect of pain relief still existed after follow-ups ranging from 3 to 8 weeks. After three weeks' follow-up, the ES of -1.60 in Lam and Cheing's study $^{22}$ was larger than the ES obtained by Vasseljen et al. ${ }^{26}$ The pooled ES after being weighted was -1.05 , which was considered to be a large effect ( $\mid$ pooled ES $\mid>0.8$ ). The 95\% CI did not include zero $(-1.16 \sim-0.94)$ and indicated a statistically significant effect $(p<0.05)$.

\section{The effects of LLLT on grasp force}

As shown in Table 7, the ESs of LLLT on grasp force were also obtained from the results of three studies with $119 \mathrm{sub}$ jects. ${ }^{21,22,26}$ Grasp force increased immediately after treat- 
Table 5. Effect Sizes of Vas after Treatment

\begin{tabular}{llccccc}
\hline Author & \multicolumn{1}{c}{ Assessable items } & $\mathrm{n}$ & Pooled SD & Wt & ES & $95 \%$ CI \\
\hline Stergioulas $^{21}$ & VAS (resting) & 50 & 6.98 & 0.16 & -0.19 & $-0.47 \sim 0.09$ \\
& VAS (pressured) & 50 & 17.43 & 0.16 & -0.92 & $-1.20 \sim-0.64^{*}$ \\
& VAS (wrist extension) & 50 & 15.64 & 0.16 & -0.79 & $-1.07 \sim-0.51^{*}$ \\
& VAS (middle finger extension) & 50 & 15.01 & 0.16 & -0.99 & $-1.27 \sim-0.72^{*}$ \\
Lam $^{22}$ & VAS (grasping) & 50 & 9.95 & 0.16 & -0.53 & $-0.81 \sim-0.25^{*}$ \\
Vasseljen $^{26}$ & VAS & 39 & 1.94 & 0.12 & -1.21 & $-1.52 \sim-0.89^{*}$ \\
Pooled & VAS & 30 & 0.68 & 0.08 & -0.28 & $-0.63 \sim 0.08$ \\
& & & & & -0.71 & $-0.82 \sim-0.60^{*}$ \\
\hline
\end{tabular}

VAS, visual analogue scale; n, numbers of patients; SD, standard deviation; Wt, weight value; ES, effect size; CI, confidence interval; *, There was a statistically significant difference.

ments and follow-up. After being weighted, the pooled ES of 0.70 was considered to represent a medium effect $(0.8>$ pooled ES $\mid>0.5)$. The $95 \%$ CI $(0.52 \sim 0.88)$ did not include zero, which indicated a statistically significant effect $(p<0.05)$. As shown in Table 8, there were significant effects on grasp force after a period of follow-up ranging from 3 to 8 weeks. Furthermore, the pooled ES of the three studies was 1.09 , which was considered to represent a large effect (|pooled $\mathrm{ES} \mid>0.8)$. The $95 \% \mathrm{CI}$ did not include zero $(0.91 \sim 1.27)$, which indicated a statistically significant effect $(p<0.05)$.

\section{The effects of LLLT on weight test}

Lam and Cheing ${ }^{22}$ did not conduct measurements of weight tests and ROM. Therefore, only the ESs of LLLT on the weight test for 80 subjects in two studies by Stergioulas ${ }^{21}$ and Vasseljen et al. ${ }^{26}$ were included and are shown in Table 7. Although there was a trend showing a favorable effect of LLLT on weight tests, Stergioulas ${ }^{21}$ did not find a statistically significant effect (95\% CI: $-0.14 \sim 0.42, p>0.05)$. After being weighted, however, the pooled ES was 0.58 , which was considered to represent a medium effect $(0.8>$ |pooled ES $\mid>0.5)$. The $95 \%$ CI $(0.37 \sim 0.80)$ did not include zero, which indicated a statistically significant effect $(p<0.05)$. After a period of follow-up ranging from 4 to 8 weeks, there were favorable and significant effects on the weight test in both studies, ${ }^{21,26}$ as shown in Table 8 . The pooled ES was 0.55 , which was considered to represent a medium effect $(0.8>\mid$ pooled ES $\mid>0.5)$. The $95 \% \mathrm{CI}$ did not include zero $(0.33 \sim 0.76)$ and indicated a statistically significant effect $(p<0.05)$

\section{The effects of LLLT on increasing ROM of the wrist}

For the 80 subjects in two studies, ${ }^{21,26}$ there was a trend of less favorable effect of LLLT on increasing ROM, as shown in Table 7, and the pooled ES was not statistically significant (pooled ES: 1.27, 95\% CI: $0.37 \sim 0.81, p<0.05$ ). However, after a period of follow-up ranging from 4 to 8 weeks, there was a trend of favorable effect in terms of increasing ROM, as depicted in Table 8. The pooled ES was 0.72 and the 95\% CI was 0.50 to 0.94 , which both showed statistically significant effects $(p<0.05)$.

\section{Discussion}

Low level laser used in clinical practice normally produces power less than $500 \mathrm{~mW}$, and can be classified as 3B according to the guidelines from the American National Standards Institute (ANSI). ${ }^{5}$ As LLLT is not based on thermal effects but on the principles of photobiomodulation, a 3B laser could reduce the dangers of burning skin, but still might harm eyes. ${ }^{21}$ Review of the four papers revealed that the application of LLLT to LE in TCM differed from application in Western medicine. ${ }^{27-30}$

\section{The effectiveness of LLLT on acupuncture points in LE}

According to evidence provided in the series of studies by Haker and Lundeberg 28,29 and Lundeberg et al., ${ }^{30}$ the application of LLLT on acupuncture points was not effective (see Table 4). In 1991, Haker and Lundeberg ${ }^{28}$ compared the study by Haker and Lundeberg ${ }^{29}$ in 1990 with the study by Lundeberg et $\mathrm{al}^{30}$ in 1987. The former had utilized lasers

Table 6. Effect Sizes of Vas after Follow-Up

\begin{tabular}{llccccc}
\hline Author & \multicolumn{1}{c}{ Assessable items } & $\mathrm{n}$ & Pooled SD & Wt & ES & $95 \%$ CI \\
\hline Stergioulas $^{21}$ (8wks) & VAS (resting) & 50 & 3.21 & 0.16 & -0.41 & $-0.69 \sim-0.13^{*}$ \\
& VAS (pressured) & 50 & 15.34 & 0.16 & -0.87 & $-1.15 \sim-0.60^{*}$ \\
& VAS (wrist extension) & 50 & 12.74 & 0.16 & -1.27 & $-1.54 \sim-0.99^{*}$ \\
& VAS (middle finger extension) & 50 & 18.69 & 0.16 & -0.72 & $-1.00 \sim-0.44^{*}$ \\
& VAS (grasping) & 50 & 8.56 & 0.16 & -1.57 & $-1.85 \sim-1.30^{*}$ \\
Lam $^{22}(3 w k s)$ & VAS & 39 & 1.75 & 0.12 & -1.60 & $-1.92 \sim-1.29^{*}$ \\
Vasseljen $^{26}(3 \mathrm{~m})$ & VAS & 30 & 0.52 & 0.08 & -1.00 & $-1.36 \sim-0.64^{*}$ \\
Pooled & & & & & -1.05 & $-1.16 \sim-0.94^{*}$ \\
\hline
\end{tabular}

*, There was a statistically significant difference. 
Table 7. Effect Sizes of Grasp Force, Weight Test, and Painless Rom after Treatment

\begin{tabular}{|c|c|c|c|c|c|c|}
\hline Author & Assessable items & $\mathrm{n}$ & Pooled SD & $W t$ & ES & $95 \% C I$ \\
\hline Stergioulas ${ }^{21}$ & grasp force & 50 & 8.93 & 0.42 & 0.88 & $0.60 \sim 1.16^{*}$ \\
\hline $\mathrm{Lam}^{22}$ & grasp force & 39 & 8.98 & 0.33 & 0.64 & $0.32 \sim 0.95^{*}$ \\
\hline Vasseljen $^{26}$ & grasp force & 30 & 0.06 & 0.25 & 0.49 & $0.14 \sim 0.85^{*}$ \\
\hline Pooled & & & & & 0.70 & $0.52 \sim 0.88^{*}$ \\
\hline Stergioulas ${ }^{21}$ & weight test & 50 & 0.86 & 0.63 & 0.14 & $-0.14 \sim 0.42$ \\
\hline Vasseljen & weight test & 30 & 0.23 & 0.37 & 1.33 & $0.97 \sim 1.68^{*}$ \\
\hline Pooled & & & & & 0.58 & $0.37 \sim 0.80^{*}$ \\
\hline Stergioulas ${ }^{21}$ & painless ROM & 50 & 11.31 & 0.63 & 0.55 & $0.27 \sim 0.83^{*}$ \\
\hline Vasseljen $^{26}$ & painless ROM & 30 & 1.20 & 0.37 & 2.50 & $2.14 \sim 2.86^{*}$ \\
\hline Pooled & & & & & 1.27 & $0.37 \sim 0.81^{*}$ \\
\hline
\end{tabular}

*, There was a statistically significant difference.

with an average power of $0.07 \mathrm{~mW}$ and energy of $0.004 \mathrm{~J} /$ point on five acupuncture points for 60 seconds per point. The latter had used lasers with an average power of $12 \mathrm{~mW}$ and energy of $0.36 \mathrm{~J} /$ point on ten acupuncture points for 30 seconds per point. Haker and Lundeberg ${ }^{28}$ found that a dose of $0.36 \mathrm{~J} /$ point would be more suitable for laser acupuncture, and that increasing the duration of laser irradiation would not guarantee better effects. Some other studies, with follow-up assessments, of subjects receiving laser acupuncture revealed that painful symptoms were consistent or worse after the treatments. ${ }^{29}$ Given the nature of local inflammation in LE, Haker and Lundeberg ${ }^{29}$ thought that the anti-inflammatory mechanism of LLLT on acupuncture points was based on photobiomodulation, which followed the Arndt-Schultz law. During needling acupuncture, pulses of mechanical stimulation can penetrate through connective tissues and activate C fibers. In TCM theory, the pulses induced by stimulating the acupuncture points can regulate the flow of qi. In dialectical thinking of TCM, LE would be diagnosed as a heat impediment or cold impediment, and approached by distinct acupuncture points. Haker and Lundeberg $^{29}$ had proposed using a HeNe laser of $632.8 \mathrm{~nm}$ and a GaAs laser of $904 \mathrm{~nm}$, capable of penetratting $0.62 \mathrm{~nm}$ and $1.4 \mathrm{~nm}$, respectively. Laser irradiance possesses a photoelectric effect and thus differs from needling acupuncture. Therefore, it remains questionable whether the depth of penetration or the physical effect of such laser irradiance is capable of attaining the effectiveness of needling acupuncture. Furthermore, the mechanical stimulation of LLLT may not be as strong as acupuncture. Therefore, the "hyperstimulation analgesia" proposed as a possible mechanism of acupuncture may not apply to LLLT. ${ }^{34}$

In summary, the effects of laser acupuncture on LE were inconsistent with those of needling acupuncture, and improvements after treatment were not obvious. Thus, based on the guidelines from the Philadelphia Panel Classification System, ${ }^{33}$ the grade of recommendation with regard to "the effectiveness of LLLT on acupuncture points in LE" was C.

\section{The effectiveness of LLLT on tender points and MTrPs in LE}

As Lam and Cheing ${ }^{22}$ indicated that injured muscles and tendons were supposed to be the target tissues in managing LE of the elbow, laser irradiation applied to tender points and MTrPs should be a better treatment option. LLLT on acupuncture points did not irradiate the areas of injured tissues, and therefore the therapeutic effects were not found. ${ }^{29,30}$ Irradiating directly on areas of tendons could activate fibroblasts to enhance the repair of damaged tissues and was thus thought to be the most appropriate and effective method of irradiation. ${ }^{22}$ Recent studies by Shah et al. $^{35,36}$ found evidence of increased concentrations of inflammatory substances in the MTrPs region. Therefore, the anti-inflammatory effect of LLLT may be the major mechanism for the relief of pain from MTrPs. After reviewing seven papers as shown in Table 2 and Table $3,{ }^{21-27}$ we found that, in the studies by Sergioulas, ${ }^{21}$ Lam and Che-

Table 8. Effect Sizes of Grasp Force, Weight Test, and Painless Rom after Follow-up

\begin{tabular}{|c|c|c|c|c|c|c|}
\hline Author & Assessable items & $\mathrm{n}$ & Pooled SD & $W t$ & ES & $95 \% C I$ \\
\hline Stergioulas $^{21}$ (8 wks) & grasp force & 50 & 9.74 & 0.42 & 1.12 & $0.84 \sim 1.40^{*}$ \\
\hline $\mathrm{Lam}^{22}(3 \mathrm{wks})$ & grasp force & 39 & 9.31 & 0.33 & 0.86 & $0.54 \sim 1.17^{*}$ \\
\hline Vasseljen $^{26}(3 \mathrm{~m})$ & grasp force & 30 & 0.06 & 0.25 & 1.33 & $0.97 \sim 1.69^{*}$ \\
\hline Pooled & & & & & 1.09 & $0.91 \sim 1.27^{*}$ \\
\hline Stergioulas $^{21}(8 \mathrm{wks})$ & weight test & 50 & 1.18 & 0.63 & 0.35 & $0.07 \sim 0.62^{*}$ \\
\hline Vasseljen $^{26}(3 \mathrm{~m})$ & weight test & 30 & 0.57 & 0.37 & 0.88 & $0.52 \sim 1.24^{*}$ \\
\hline Pooled & & & & & 0.55 & $0.33 \sim 0.76^{*}$ \\
\hline Stergioulas ${ }^{21}$ (8 wks) & painless ROM & 50 & 11.93 & 0.63 & 0.66 & $0.38 \sim 0.93^{*}$ \\
\hline Vasseljen $^{26}(3 \mathrm{~m})$ & painless ROM & 30 & 1.20 & 0.37 & 0.83 & $0.48 \sim 1.19^{*}$ \\
\hline Pooled & & & & & 0.72 & $0.50 \sim 0.94^{*}$ \\
\hline
\end{tabular}

*, There was a statistically significant difference. 
ing, ${ }^{22}$ and Vasseljen et al., ${ }^{26}$ not only was pain reduced statistically $(p<0.05)$ after LLLT, but also grasp force and performance during functional movements were improved statistically $(p<0.05)$. Lam and Cheing ${ }^{22}$ proposed that pain relief was related to the increased metabolism of adenosine triphosphate (ATP) in mitochondria, which resulted in the increased metabolism of MTrPs. ${ }^{22}$ Hence, the focus on MTrPs for the treatment of LE could be more effective through induced local hypoxia and improvement of blood circulation. Even so, Krasheninnikoff et al. ${ }^{25}$ had found no statistically significant effectiveness of LLLT on tender points in LE $(p>0.05)$. Because appropriate parameters and doses of laser were unclear, they also proposed that applying LLLT to musculoskeletal diseases might also be ineffective.

To summarize, the treatment mechanisms were consistent and the effectiveness of different treatments was statistically significant in some studies $(p<0.05),{ }^{21,22,26}$ but other studies did not find statistically significant improvement $(p>0.05){ }^{23-25,27}$ Therefore, based on the guidelines from the Philadelphia Panel Classification System, ${ }^{33}$ the grade of recommendation with regard to "the effectiveness of LLLT on tender points and MTrPs in LE in Western medicine"was C+ to $\mathrm{B}$.

\section{Appropriate treatment parameters of $L L L T$ for $L E$}

The photobiomodulation of LLLT was related to three parameters: wavelength, energy density, and power, all three of which were crucial in determining the effectiveness of LLLT. ${ }^{21}$ Energy density was the energy absorbed by superficial tissues, usually in terms of $\mathrm{J} / \mathrm{cm}^{2}$, and was referred to as treatment dosage. Sergioulas ${ }^{21}$ suggested that using a laser of $904 \mathrm{~nm}$ wavelength with an average power of $40 \mathrm{~mW}$ should be appropriate for musculoskeletal diseases. Lam and Cheing ${ }^{22}$ indicated that the depth of penetration was another important factor, and a laser with a wavelength of $920 \mathrm{~nm}$, which is almost the wavelength of visible red light, had better penetration ability. They also reported that laser with pulsed output could produce better penetration effects than continuous wave laser. This was due to lowered impedance across the skin, by which $37 \%$ of the laser energy would otherwise be blocked. ${ }^{29}$ In a series of studies by Haker and Lundeberg $^{27-29}$ and Lundeberg et al. ${ }^{30}$ in 1987, a double wavelength laser combining $940 \mathrm{~nm}$ and $632.8 \mathrm{~nm}$ was utilized as a tool of laser acupuncture. ${ }^{30}$ In 1991, Haker and Lundeberg ${ }^{29}$ used a $940 \mathrm{~nm}$ probe laser to treat tender points combined with a pen-point laser to irradiate on acupuncture points. ${ }^{28}$ However, the effects seen in these studies did not reach statistical significance $(p>0.05)$. Sergioulas ${ }^{21}$ inferred that the different doses and types of LLLT used in this series of studies had led to the ineffectiveness. Lam and Cheing ${ }^{22}$ also thought that the doses used in this series of studies were insufficient. Addressing the issue of lower energy used in those studies, Krasheninnikoff et al. ${ }^{25}$ decided on utilizing a laser with more energy (3.6 J/point) than the $0.004 \mathrm{~J} /$ point used in the Lundeberg et al. study ${ }^{30}$ and the $0.36 \mathrm{~J} /$ point in the Haker and Lundeberg study. ${ }^{27,29}$ Two of the studies used laser on tender points, ${ }^{25,27}$ while the others used laser on acupuncture points. ${ }^{29,30}$ Krasheninnikoff et al., ${ }^{25}$ however, observed no statistically significant differences $(p>0.05)$, and instead found results that were similar to those of the others. It seems that the energy density of LLLT must be accentuated and canvassed. We found a lack of evidence that could be used to ascertain the appropriate treatment dose for laser acupuncture. Regarding treatment dose on MTrPs, Lam and Cheing 22 showed that energy density ranging from 0.3 to $3 \mathrm{~J} / \mathrm{cm}^{2}$ was thought to be appropriate for LE. Basford et al. ${ }^{23}$ had tried a longer wavelength $(1.06 \mu \mathrm{m})$ with stronger treatment dosage $\left(12.24 \mathrm{~J} / \mathrm{cm}^{2}\right)$ on tender points, and a larger probe (radius: $5 \mathrm{~cm}$ ) to increase the irradiance. No statistically significant effectiveness, however, was observed in the parameters of pain and grasp force $(p>0.05)$. Sergioulas ${ }^{21}$ indicated that LLLT with wavelength of $904 \mathrm{~nm}$, average power of $40 \mathrm{~nm}$, frequency of $60 \mathrm{~Hz}$, and dosage of $2.4 \mathrm{~J} / \mathrm{cm}^{2}$ on tender points was most effective for LE $(p<0.05)$. These parameters were also suggested for the treatment of other musculotendinous diseases. ${ }^{21}$ Therefore, an energy density of $2.4 \mathrm{~J} / \mathrm{cm}^{2}$ on tender points was presumed to be an appropriate treatment dose, and energy that was stronger would be ineffective. More studies with RCT are required to provide stronger evidence for the effectiveness of this dose.

\section{Improvement in pain relief, grasp force, ROM, and weight test}

The results of our meta-analysis showed that using LLLT for LE exerted statistically significant effects in the areas of pain relief, increasing grasp force, increasing ROM of wrist joints, and improving weight tests, regardless of whether the testing was conducted after the treatments or after the follow-up visits $(p<0.05)$. After separately reviewing the results of Sergioulas ${ }^{21}$ and Lam and Cheing, ${ }^{22}$ we concluded that "using LLLT in LE can reduce pain significantly", and the grade of recommendation would be A. Lam and Cheing $^{22}$ thought that the analgesia mechanism for LLLT was dependent on an immediate decrease in the synthesis of prostaglandins and the conduction velocities of $\mathrm{A} \delta$ and $\mathrm{C}$ fibers. They also suggested that the absorption of LLLT could be accumulated during the treatment sessions. As a result, they conducted LLLT three times per week, and did not find any obvious improvement in PPT $(p>0.05)$ until the fifth treatment $(p<0.05)$. However, these effects remained for only three weeks after treatment. Vasseljen et al. ${ }^{26}$ also found that $47 \%$ of subjects had improvement in symptoms after four weeks' follow-up.

Measures of grasp force, ROM of wrist joints, and weight tests can illustrate functional improvement in symptoms and recovery of muscle strength. According to the analyses of Sergioulas, ${ }^{21}$ Lam and Cheing, ${ }^{22}$ and Vasseljen et al., ${ }^{26}$ there was statistically significant improvement after treatment and follow-up $(p<0.05)$. To review separately the results of these studies, ${ }^{21,22,26}$ the grade of recommendation for the statement "using LLLT in LE could improve muscle strength significantly" would be A. The study by Sergioulas ${ }^{21}$ involved plyometrics training for both experimental and control groups. The training program included pain-free wrist extension movements which progressed from active to resistant, to be done twice a week for 16 weeks. Lam and Cheing $^{22}$ also conducted a 3-week exercise program that included stretching and strengthening exercises of the forearm for both experimental and control groups. These exercises were considered to have positive effects for clinical application. 


\section{Our suggestions and limitations}

Many assessment measurements have been used to manage LE, but some of the assessment tools were considered to be inappropriate after reviewing some studies. ${ }^{25,27,30}$ Haker and Lundeberg ${ }^{27}$ and Lundeberg et al. ${ }^{30}$ thought that FPV could not be utilized to detect and assess minor changes in pain reduction, and that the MMT rating system for muscle strength, with six grades, also had this problem. ${ }^{25}$ Highly sensitive measurement tools were necessary. Vasseljen et $\mathrm{al}^{26}$ suggested that the VAS, grip dynamometer, and weight tests were better assessment tools for LE. Due to the lower number of RCTs in laser acupuncture, we were unable to further explore the effectiveness of LLLT on acupuncture points for LE. Moreover, we suggest that more stringent RCTs of TCM are needed to compare its therapeutic effects with Western medicine.

This study had some limitations in understanding the effectiveness of applying LLLT for LE. Only three papers could be used for the meta-analysis ${ }^{21,22,26}$ because there were no unified standards for various assessment tools in the papers we had collected. There were no detailed values for the assessments after treatment and follow-up which could have been used for further meta-analysis.

\section{Conclusion and Summary}

Few articles in the literature address the use of LLLT on acupuncture points in TCM-based treatment. Tender points in LE were diagnosed as part of the Western medicine treatment process, but Ashi points were not. Therefore, the current evidence justifying the therapeutic effects of LLLT on LE in Western medicine was better than that for TCM. More exact diagnosis of Ashi points and clinical RCTs will be needed to prove the effects of LLLT in TCM. LLLT on tender points and MTrPs would be more appropriate. There were no consistent conclusion as to the appropriate parameters of LLLT, and more studies will be needed.

\section{Acknowledgments}

The authors are grateful to the National Science Council of the Republic of China for financially supporting this research under contracts no. NSC 96-2218-E-002-015, NSC 97-2218-E002-006, and NSC 96-2628-E-002-252-MY3.

\section{Disclosure Statement}

\section{References}

1. Calfee, R.P., Patel, A., DaSilva, M.F., and Akelman, E. (2008) Management of lateral epicondylitis: current concepts. J. Am. Acad. Orthop. Surg. 16, 19-29.

2. Shiri, R., Viikari-Juntura, E., Varonen, H., and Heliövaara, M. (2006). Prevalence and determinants of lateral and medial epicondylitis: a population study. Am. J. Epidemiol. 164, 1065-1074.

3. Smidt, N., Lewis, M., van der Windt, D.A., Hay, E.M., Bouter, L.M., and Croft, P. (2006). Lateral epicondylitis in general practice: course and prognostic indicators of outcome. J. Rheumatol. 33, 2053-2059.
4. Trinh, K.V., Phillips, S.D., Ho, E., and Damsma, K. (2004). Acupuncture for the alleviation of lateral epicondyle pain: a systematic review. Rheumatology 43, 1085-1090.

5. Viola, L. (1998). A critical review of the current conservative therapies for tennis elbow (lateral epicondylitis). Australas. Chiropr. Osteopathy 7, 53-67.

6. Melzack, R., Stillwell, D.M., and Fox, E.J. (1997). Trigger points and acupuncture points for pain: correlations and implications. Pain 3, 3-23.

7. Hong, C.Z., and Simons, D.G. (1998). Pathophysiologic and electrophysiologic mechanisms of myofascial trigger points. Arch. Phys. Med. Rehabil. 79, 863-872.

8. Hong, C.Z. (2000). Myofascial trigger points: pathophysiology and correlation with acupuncture points. Acup. Med. $18,41-47$

9. Xu, R.D., and Li, H. (2005). Conception of Ashi points. Zhongguo Zhen Jiu 25, 281-283.

10. Simons, D.G., Travell, J.G., and Simons, L.S. (1999). Travell \& Simons's Myofascial Pain and Dysfunction: The Trigger Point Manual. 2nd ed. Baltimore (MD): Williams \& Wilkins, pp. 94-173.

11. Schjelderup, V. (1984). The use of laser therapy in acupuncture. Acupunct. Med. 2, 22-27.

12. Hecox, B., Andemicael Mehreteab, T., and Weisberg, J. (1994). Physical Agents: A Comprehensive Text for Physical Therapists. Norwalk (CT): Appleton \& Lange, pp. 391-396.

13. Chang, W.D., Wu, J.H., Jiang, J.A., Yeh, C.Y., and Tsai, C.T. (2008). Carpal tunnel syndrome treated with a diode laser: a controlled treatment of the transverse carpal ligament. Photomed. Laser Surg. 26, 551-557.

14. Tam, G. (1999). Low power laser therapy and analgesic action. J. Clin. Laser Med. Surg. 17, 29-33.

15. Ohshiro, T. (1988). Low Level Laser Therapy. Avon (U.K.): Wiley and Sons, pp. 16-30.

16. Ghamsari, S.M., Acorda, J.A., Taguchi, K., Abe, N., and Yamada, H. (1996). Evaluation of wound healing of the teat with and without low level laser therapy in dairy cattle by laser Doppler flowmetry in comparison with histopathology, tensiometry and hydroxyproline analysis. Br. Vet. J. 152, 583-592.

17. Houreld, N.N., and Abrahamse, H. (2008). Laser light influences cellular viability and proliferation in diabeticwounded fibroblast cells in a dose- and wavelengthdependent manner. Lasers Med. Sci. 23, 11-18.

18. van Breugel, H.H., and Bär, P.R. (1992). Power density and exposure time of He-Ne laser irradiation are more important than total energy dose in photo-biomodulation of human fibroblasts in vitro. Lasers Surg. Med. 12, 528-537.

19. Bjordal, J.M., Lopes-Martins, R.A., Joensen, J., et al. (2008). A systematic review with procedural assessments and metaanalysis of low level laser therapy in lateral elbow tendinopathy (tennis elbow). B.M.C. Musculoskelet. Disord. 9, 75.

20. Stasinopoulos, D.I., and Johnson, M.I. (2005). Effectiveness of low-level laser therapy for lateral elbow tendinopathy. Photomed. Laser Surg. 23, 425-430.

21. Stergioulas, A. (2007). Effects of low-level laser and plyometric exercises in the treatment of lateral epicondylitis. Photomed. Laser Surg. 25, 205-213.

22. Lam, L.K., and Cheing, G.L. (2007). Effects of 904-nm low-level laser therapy in the management of lateral epicondylitis: a randomized controlled trial. Photomed. Laser Surg. 25, 65-71.

23. Basford, J.R., Sheffield, C.G., and Cieslak, K.R. (2000). Laser therapy: a randomized, controlled trial of the effects of low intensity Nd:YAG laser irradiation on lateral epicondylitis. Arch. Phys. Med. Rehabil. 81, 1504-1510. 
24. Papadopoulos, E.S., and Smith, R.W. (1996). Low-level laser therapy does not aid the management of tennis elbow. Clin. Rehabil. 10, 9-11.

25. Krasheninnikoff, M., Ellitsgaard, N., Rogvi-Hansen, B., et al. (1994). No effect of low power laser in lateral epicondylitis. Scand. J. Rheumatol. 23, 260-263.

26. Vasseljen, O. Jr., Høeg, N., Kjeldstad, B., Johnsson, A., and Larsen S. (1992). Low level laser versus placebo in the treatment of tennis elbow. Scand. J. Rehabil. Med. 24, 37-42.

27. Haker, E., and Lundeberg, T. (1991). Is low-energy laser treatment effective in lateral epicondylalgia? J. Pain Symptom Manage. 6:241-246.

28. Haker, E., and Lundeberg, T. (1991). Lateral epicondylalgia: report of noneffective midlaser treatment. Arch. Phys. Med. Rehabil. 72, 984-988.

29. Haker, E., and Lundeberg, T. (1990). Laser treatment applied to acupuncture points in lateral humeral epicondylalgia: A double-blind study. Pain 43, 243-247.

30. Lundeberg, T., Haker, E., and Thomas, M. (1987). Effect of laser versus placebo in tennis elbow. Scand. J. Rehabil. Med. 19, 135-138.

31. Moseley, A.M., Herbert, R.D., Sherrington, C., and Maher, C.G. (2002). Evidence for physiotherapy practice: a survey of the Physiotherapy Evidence Database (PEDro). Aust. J. Physiother. 48, 43-49.

32. Egger, M., and Smith, G.D. (1997). Meta-Analysis. Potentials and promise. B.M.J. 315, 1371-1374.
33. Harris, G.R., and Susman, J.L. (2002). Managing musculoskeletal complaints with rehabilitation therapy: summary of the Philadelphia Panel evidence-based clinical practice guidelines on musculoskeletal rehabilitation interventions. J. Fam. Pract. 51, 1042-1046.

34. Melzack, R. (1981). Myofascial trigger points: relation to acupuncture and mechanism of pain. Arch. Phys. Med. Rehabil. 62, 114-117.

35. Shah, J.P., Danoff, J.V., Desai, M.J., et al. (2008). Biochemicals associated with pain and inflammation are elevated in sites near to and remote from active myofascial trigger points. Arch. Phys. Med. Rehabil. 89, 16-23.

36. Shah, J.P. (2008). Uncovering the biochemical milieu of myofascial trigger points. Using in vivo microdialysis. J. Musculoske. Pain 16, 17-20.

Address correspondence to: Joe-Air Jiang, Ph.D., P.Eng. Department of Bio-Industrial Mechatronics Engineering National Taiwan University No. 1, Sec. 4, Roosevelt Road Taipei 10617 Taiwan

E-mail: jajiang@ntu.edu.tw 


\section{AUTHOR QUERY FOR PHO-2009-2558-CHANG_1P}

AU1: Please enter a financial disclosure statement. If no potential conflicts of interest exist, please enter "No competing financial interests exist."

AU2: Please include cities for these affiliations.

AU3: Please provide academic titles (e.g., Ph.D.) for each author.

AU4: Please spell out this abbreviation.

AU5: Meaning unclear; please clarify.

AU6: Unconventional way of writing these. Please confirm that you want to express these values this way. 